\title{
Screening of COVID-19 in outpatient children with cancer or solid organ transplantation: preliminary report
}

\author{
Thaís Lira Cleto-Yamane ${ }^{1,2}$ (1) - Gustavo Rodrigues-Santos ${ }^{3} \cdot$ Maria Clara de Magalhães-Barbosa $^{3}$. \\ Patrícia Gomes Moura ${ }^{4}$. Rafael Dias Vasconcelos ${ }^{5}$. Jaqueline Leal Santos Gouveia ${ }^{1,6}$ • Anne Louise de Oliveira ${ }^{1,6}$. \\ Fernanda Couto Ferreira ${ }^{5}$. Ana Letícia Shalders ${ }^{4}$ - Mariana Barros Genuíno de Oliveira ${ }^{3}$. Fernanda Lima-Setta ${ }^{3}$. \\ Antonio José Ledo Alves da Cunha ${ }^{3,7}$. Arnaldo Prata-Barbosa ${ }^{3,7}$
}

Received: 2 December 2020 / Revised: 10 March 2021 / Accepted: 21 March 2021 / Published online: 26 March 2021

(C) The Author(s), under exclusive licence to Springer-Verlag GmbH Germany, part of Springer Nature 2021

\begin{abstract}
Clinical presentation of severe acute respiratory syndrome coronavirus 2 (SARS-CoV-2) infection in pediatric immunosuppressed patients is unknown. Emerging data describe a milder or asymptomatic course in children compared with adults in this scenario. We present the seroprevalence and clinical features of coronavirus disease 2019 in a prospective cohort of 114 immunosuppressed children and adolescents from three groups: kidney transplantation, liver transplantation, and cancer patients. Among the thirty-five $(30.7 \%)$ patients who had a positive serological test for SARS-CoV-2, 77\% did not report previous symptoms and none of them developed any complications of coronavirus disease 2019 (COVID-19) after 30 or more days of follow-up. Among those who were symptomatic, diarrhea, fever, and cough were the most common findings.

Conclusion: Seroprevalence of SARS-CoV-2 infection is high among immunosuppressed children and adolescents. COVID19 has a mild or asymptomatic course in most of these patients.
\end{abstract}

\section{What is Known:}

- The number of immunosuppressed patients with coronavirus disease 2019 is increasing.

- Viral infections have the potential for greater severity in immunocompromised children.

What is New:

- Seroprevalence for severe acute respiratory syndrome coronavirus 2 in immunocompromised pediatric patients was $31 \%$.

- A quarter of the serology-positive patients reported mild symptoms and none of them developed multisystem inflammatory syndrome in children associated with coronavirus disease 2019.

Communicated by Nicole Ritz

Thaís Lira Cleto-Yamane

thaiscleto@yahoo.com.br

Gustavo Rodrigues-Santos

gustavorodriguesrj@gmail.com

\author{
Maria Clara de Magalhães-Barbosa \\ mariaclaramb@globo.com \\ Patrícia Gomes Moura \\ patriciagmoura@globo.com \\ Rafael Dias Vasconcelos \\ rafaelvasconceloscirurgia@gmail.com \\ Jaqueline Leal Santos Gouveia \\ jaqueleal@yahoo.com.br \\ Anne Louise de Oliveira \\ anne_louise03@yahoo.com.br
}

Fernanda Couto Ferreira

fcoutof@yahoo.com.br

Ana Letícia Shalders

analeticia.shalders@hotmail.com

Mariana Barros Genuíno de Oliveira

mgenuino@gmail.com

Fernanda Lima-Setta

felimasetta@gmail.com

Antonio José Ledo Alves da Cunha antonioledo@yahoo.com.br

Arnaldo Prata-Barbosa

arnaldoprata@globo.com

Extended author information available on the last page of the article 
Keywords SARS-CoV-2 $\cdot$ COVID-19 $\cdot$ Pediatrics $\cdot$ Liver transplantation $\cdot$ Kidney transplantation $\cdot$ Leukemia $\cdot$ Oncology

\section{Introduction}

Viral infections are frequent in children and have the potential for greater severity in immunocompromised patients [1]. Clinical presentation of severe acute respiratory syndrome caused by the new coronavirus (SARS-CoV-2) in pediatric immunosuppressed patients is still unknown. Immunosuppressive drugs alter the patient's immune response, acting on humoral, cellular immunity, and neutrophil function, increasing the risk of serious viral infections [1-3]. Severe cases of COVID-19 have been associated with an imbalance between the inflammatory system and the immune system which determine an exacerbated reaction with the release of pro-inflammatory and anti-inflammatory cytokines. Such fact can generate "cytokine storm syndrome," resulting in multiple organ dysfunction syndrome [4].

The March 2020 report from the Papa Giovanni XXIII Hospital in Bergamo, a major liver transplant center in Italy, showed that the number of transplant patients infected with COVID-19 increased steadily, but they did not see greater severity and complications in this group of patients compared with the general population [5]. A survey that included 18 kidney transplant patients aged 0-19 years with COVID-19 in 11 countries described a mild clinical course in this specific group of patients [6].

The present study aims to describe our preliminary results on the prevalence of viral infection by SARS-CoV-2 in a cohort of immunosuppressed children and adolescents from three groups: kidney transplantation, liver transplantation, and cancer patients. We also analyzed the epidemiological profile and clinical evolution of these patients. Our hypothesis is that immunosuppression may act as protection for symptomatic presentations, especially for severe forms of COVID-19.

\section{Materials and methods}

This is a prospective study of a cohort of immunosuppressed patients, from 0 to 18 years old, in a tertiary hospital in Rio de Janeiro, Brazil. All patients who were being followed up at the hospital at the beginning of the COVID-19 pandemic and who came for follow-up appointments after kidney or liver transplantation, or during cancer treatment, from June 30 to August 28,2020 were included in this preliminary report. After agreeing to participate by signing an informed consent form, all patients were tested for the presence of $\mathrm{IgM} / \mathrm{IgG}$ antibodies against SARS-CoV-2 using a rapid colloidal gold immunochromatography test (Beijing LEPU-Medical Technology). Patient and caregivers were asked about any symptoms suggestive of COVID-19 and about contact with a suspected case. The electronic medical records of each patient were also reviewed for demographic, clinical, and laboratory data.

In the first 2 weeks of the study, the RT-PCR test for SARS-CoV-2 was only available to patients admitted for treatment of COVID-19. From the third week on, it became available and was collected from all IgM-positive and/or symptomatic. From those, a nasopharyngeal swab was collected and the viral RNA extracted with QIAamp viral RNA kit (Qiagen) and submitted to real-time RT-PCR to detect the SARS-CoV-2 E gene (Charité protocol, Berlin, Germany), and targets N1 and N2 (CDC protocol, USA). Any return to hospital and/or hospitalization for SARS-CoV-2 infection or occurrence of multisystem inflammatory syndrome in children (MIS-C) was recorded. On continuity of the study, all patients will be followed up at the hospital and repeat the rapid serological test (IgM/IgG antibodies against SARS-CoV-2) 6 months after the first test. The study was approved by the institution's research ethics committee and is registered in ClinicalTrials.gov (protocol ID: NCT04511429).

\section{Results}

From the 115 eligible patients, 114 were included (1 refused to participate): 72 transplant patients (69.4\% kidney, 30.6\% liver) and 42 undergoing cancer treatment $(54.8 \%$ with acute lymphoblastic leukemia). The median age was 11 years, there was no gender predominance, and the majority were nonwhites $(65 \%)$. Median follow-up time from transplant to the day of the test was 866 days. Among cancer patients, the median time between the start of chemotherapy and the test was 238 days. All transplant patients were receiving immunosuppressive drugs ( $84 \%$ were under 2 or more drugs). Of the group of cancer patients, the majority $(92.8 \%, n=39)$ were undergoing chemotherapy, 6 of which were in the induction phase and 7 in the maintenance phase. Only 3 patients of this group were in the control phase, all of them had received chemotherapy until 15 days to 2 months before the test. The most common drug among transplant patients was tacrolimus, followed by prednisone and mycophenolate sodium. In the cancer group, most patients were treated with AEIOP-BFMALL Protocol 2009 version 2013 and ALL-BFM-IC 2009. One patient in the kidney transplant group had received 
Table 1 Contact history. clinical features, and diagnosis confirmation of the cohort $(n=114)$

\begin{tabular}{|c|c|c|c|c|}
\hline Characteristic & Total $(n=114)$ & Kidney transplantation $(n=50)$ & Liver transplantation $(n=22)$ & Cancer $(n=42)$ \\
\hline Contact with a suspect case & $24(21.1)$ & $14(28)$ & $3(13.6)$ & $7(16.7)$ \\
\hline Household & $22(19.3)$ & $12(85.7)$ & $3(100)$ & $7(100)$ \\
\hline Other & $2(1.8)$ & $2(14.3)$ & $0(0)$ & $0(0)$ \\
\hline \multicolumn{5}{|c|}{ SARS-CoV-2 infection by positive serology $(n=35)$} \\
\hline Positive serology & $35(30.7)$ & $21(42)$ & $4(18.2)$ & $10(23.8)$ \\
\hline $\operatorname{IgM}$ & $10(28.6)$ & $7(33.3)$ & $2(50)$ & $1(10)$ \\
\hline $\operatorname{IgG}$ & $15(42.8)$ & $10(47.6)$ & $1(25)$ & $4(50)$ \\
\hline $\operatorname{IgM}$ and $\operatorname{IgG}$ & $10(28.6)$ & $4(19.1)$ & $1(25)$ & $5(50)$ \\
\hline \multicolumn{5}{|c|}{ Patients with positive serology $(n=35)$} \\
\hline Asymptomatic ${ }^{\mathrm{a}}$ & $27 / 35(77.1)$ & $17 / 21(81)$ & $3 / 4(75)$ & $7 / 10(70)$ \\
\hline Symptomatic $^{\mathrm{a}}$ & $8 / 35(22.9)$ & $4 / 21(19)$ & $1 / 4(25)$ & $3 / 10(30)$ \\
\hline Contact with suspect cases ${ }^{\mathrm{a}}$ & $17 / 35(48.6)$ & $11 / 21(52.4)$ & $1 / 4(25)$ & $5 / 10(50)$ \\
\hline Contact with suspect cases and symptomatic ${ }^{\mathrm{a}}$ & $5 / 35(14.3)$ & $3 / 21(14.3)$ & $0 / 4(0)$ & $2 / 10(20)$ \\
\hline Reported signs and symptoms & $8(7)$ & $4(8)$ & $1(4.6)$ & $3(7.1)$ \\
\hline Diarrhea & $4(36.4)$ & $1(14.3)$ & $0(0)$ & $3(100)$ \\
\hline Fever & $4(36.4)$ & $3(42.9)$ & $0(0)$ & $1(33)$ \\
\hline Cough & $3(27.3)$ & $3(42.9)$ & $0(0)$ & $0(0)$ \\
\hline Neutropenia & $2(18.2)$ & $0(0)$ & $0(0)$ & $2(67)$ \\
\hline Coryza & $2(18.2)$ & $1(14.3)$ & $1(100)$ & $0(0)$ \\
\hline Anosmia & $2(18.2)$ & $2(28.6)$ & $0(0)$ & $0(0)$ \\
\hline Vomiting & $1(9.1)$ & $1(14.3)$ & $0(0)$ & $0(0)$ \\
\hline Feed refusal & $1(9.1)$ & $1(14.3)$ & $0(0)$ & $0(0)$ \\
\hline Hypotension & $1(9.1)$ & $1(14.3)$ & $0(0)$ & $0(0)$ \\
\hline Sore throat & $1(9.1)$ & $1(14.3)$ & $0(0)$ & $0(0)$ \\
\hline Headache & $1(9.1)$ & $1(14.3)$ & $0(0)$ & $0(0)$ \\
\hline Rash & $1(9.1)$ & $1(14.3)$ & $0(0)$ & $0(0)$ \\
\hline Tachypnea, $\mathrm{SpO}_{2}<92 \%$ & $0(0)$ & $0(0)$ & $0(0)$ & $0(0)$ \\
\hline Dehydration, prostration & $0(0)$ & $0(0)$ & $0(0)$ & $0(0)$ \\
\hline
\end{tabular}

$\mathrm{SpO}_{2}$ pulse oximeter oxygen saturation

${ }^{a}$ Patients with at least 1 positive test (IgM or $\operatorname{IgG}$ )

intravenous immunoglobulin (IVIG) 1 month before the test. This patient had negative IgM and IgG for SARS-CoV-2.

Twenty-four subjects $(21 \%)$ reported contact with suspected cases of COVID-19 (92\% a household contact). Thirty-five $(30.7 \%)$ patients had a positive serological test for SARS-CoV-2, the majority in the liver transplant group (Table 1). The median time in days between presentation of symptoms and the test was 9 days. RT-PCR was performed in nine serology-positive patients and was positive in only one asymptomatic IgM-positive/IgG-negative patient. Among patients with positive serology ( $n=35), 77 \%$ did not report previous symptoms and, among those who were symptomatic, diarrhea, fever, and cough were the most common findings, but none reported tachypnea or prostration (Table 1). All symptomatic patients were followed up on an outpatient basis and there was no need for hospitalization. None of the patients with a negative serological test reported previous symptoms. The seronegative patients remained asymptomatic in the period of observation.

With 30 or more days of follow-up at the time of this report, no patients developed MIS-C, was admitted to the hospital for complications from COVID-19, or even returned to the outpatient clinic due to symptoms related to SARS-CoV-2 infection. None of them died.

\section{Discussion}

In this preliminary report, we found a seroprevalence of $31 \%$ for SARS-CoV-2 infection in immunocompromised pediatric patients. Reported seroprevalence for SARS-CoV-2 infection in Rio de Janeiro in June 2020 was $7.5 \%$ [7]. Only a quarter of 
the antibody-positive patients in our cohort reported mild symptoms, which did not require medical assistance. Diarrhea was the most frequent symptom, followed by fever and cough, and no patient with negative serology reported previous symptoms.

At the time of writing this report, we have found no prospective study describing the seroprevalence and clinical features of COVID-19 in immunocompromised pediatric patients, regardless of the presence of symptoms. Since the beginning of the COVID-19 pandemic, efforts have been made to determine the characteristics of this disease in specific groups of patients. It is already known that children have a milder course of the disease and better outcomes than adults [8], and the few studies that evaluated immunosuppressed patients infected with SARS-Cov-2 did not show a more severe course of the disease as compared with general population $[5,9]$. This may be partially explained by the impaired immune response in these patients, as the severity of the disease depends in part on the host's response to infection $[5,10]$. A recent systematic review identified 16 articles on SARSCoV-2 infection in 110 immunosuppressed patients, only four of whom were children [3]. The seroprevalence in our report was about one-third and only a quarter of patients reported mild symptoms, especially diarrhea and fever, without severe respiratory symptoms. This differs from a study that included immunocompetent children with COVID-19, who had fever and cough as the main symptoms [11], and from a systematic review that described gastrointestinal symptoms in only $17 \%$ of children [9]. In our cohort, the majority of confirmed patients reported no symptoms. The frequency of asymptomatic children infected with SARS-CoV-2 in the general pediatric population is unknown. In China, $4.4 \%$ and $15.8 \%$ of asymptomatic children were found in two large cohorts of the general pediatric population with confirmed and suspected cases of COVID-19, based on clinical manifestations or history of exposure $[2,6]$. These preliminary findings suggest that immunocompromised children infected with SARS-CoV-2 are predominantly asymptomatic and that symptomatic patients have mild forms of the disease. Severe cases of COVID-19 appear to be infrequent in this population.

This study has some limitations: The main one is the sensitivity of immunoassay test used. Although the manufacturer reports a sensitivity of $98.9 \%$ and specificity of $97.6 \%$, a recent meta-analysis showed a sensitivity of $66 \%$ and a specificity of $97 \%$ for this type of test [12]. As the test used has a lower sensitivity than the test recommended by the WHO (RT-PCR), we consider that the seroprevalence for SARS-CoV-2 may be even greater than that found in this study, mainly in the group of asymptomatic patients. Another limitation is the time when the test was performed. We performed the test during the medical appointment and it had no temporal relationship with symptoms or contact with suspected cases.
Therefore, some patients may have been infected in a short period before the test and may have not yet developed antibodies to be detected by the test. But this would also further increase the prevalence found.

In conclusion, the seroprevalence of SARS-CoV-2 infection is high among immunosuppressed children and adolescents. COVID-19 has a mild or asymptomatic course in most of these patients.

Abbreviations AEIOP, Associazione Italiana Ematologia ed Oncologia Pediatrica; ALL, Acute lymphoblastic leukemia; BFM, Berlin-FrankfurtMunich; CDC, Center for Diseases Control; COVID-19, Coronavirus disease 2019; IC, Intercontinental; IVIG, Intravenous immunoglobulin; MIS-C, Multisystem inflammatory syndrome in children; RT-PCR, Reverse transcription-polymerase chain reaction; SARS-CoV-2, Severe acute respiratory syndrome coronavirus 2; WHO, World Health Organization

Acknowledgements We thank to Drs Heloisa Graça Aranha, Jason Guida do Araújo, and Lúcio Miranda de Abreu, directors of Rio de Janeiro Children`s State Hospital, for making this research possible.

Authors' contributions Dr Thaís Lira Cleto-Yamane, Dr Maria Clara de Magalhães-Barbosa, Dr Antonio José Ledo Alves da Cunha, and Dr Arnaldo Prata-Barbosa conceptualized and designed the study, coordinated the data collection, and reviewed and revised the manuscript.

Dr Gustavo Rodrigues-Santos and Dr Fernanda Lima-Setta designed the study, carried out the initial analyses, designed the data collection instruments, coordinated and supervised the data collection, and reviewed and revised the manuscript.

Dr Patrícia Gomes Moura, Dr Rafael Dias Vasconcelos, Dr Jaqueline Leal Santos Gouveia, Dr Anne Louise de Oliveira, Dr Fernanda Couto Ferreira, Dr Ana Letícia Shalders, and Mariana Barros Genuíno de Oliveira collected the data and reviewed and revised the manuscript.

All authors approved the final manuscript as submitted and agree to be accountable for all aspects of the work.

Funding This study is supported by Fundação Carlos Chagas Filho de Amparo à Pesquisa do Estado do Rio de Janeiro FAPERJ (Carlos Chagas Filho Foundation for Research Support of the State of Rio de Janeiro FAPERJ) (Process n ${ }^{\circ}$ E-26/010.000159/2020, grant ${ }^{\circ} 210.165 / 2020$ to AJLAC).

Availability of data and material Data is available in a data repository.

Code availability Not applicable.

\section{Declarations}

Ethics approval Approval was obtained from the ethics committee of D'Or Institute for Research and Education. The procedures used in this study adhere to the tenets of the Declaration of Helsinki.

Consent to participate Written informed consent was obtained from the parents.

Consent to publish Parents signed informed consent regarding publishing their children/adolescent data.

Competing interests The authors declare no competing interests. 


\section{References}

1. Kaltsas A, Sepkowitz K (2012) Community acquired respiratory and gastrointestinal viral infections: challenges in the immunocompromised host. Curr Opin Infect Dis. 25(4):423-430

2. Safdar A, Armstrong D (2011) Infections in patients with hematologic neoplasms and hematopoietic stem cell transplantation: neutropenia, humoral, and splenic defects. Clin Infect Dis. 53(8):798806

3. Dandamudi R, Smith J, Dharnidharka VR (2019) Renal transplantation and predisposition to opportunistic infections. Curr Opin Pediatr. 31(2):226-231

4. Tang Y, Liu J, Zhang D, Xu Z, Ji J, Wen C (2020) Cytokine storm in COVID-19: the current evidence and treatment strategies. Front Immunol. 11:1708

5. D'Antiga L (2020) Coronaviruses and immunosuppressed patients. The facts during the third epidemic. Liver Transpl

6. Marlais M, Wlodkowski T, Vivarelli M, Pape L, Tönshoff B, Schaefer F, Tullus K (2020) The severity of COVID-19 in children on immunosuppressive medication. Lancet Child Adolesc Health. 4(7):e17-ee8

7. Hallal PC, Hartwig FP, Horta BL, Silveira MF, Struchiner CJ, Vidaletti LP, Neumann NA, Pellanda LC, Dellagostin OA, Burattini MN, Victora GD, Menezes AMB, Barros FC, Barros AJD, Victora CG (2020) SARS-CoV-2 antibody prevalence in
Brazil: results from two successive nationwide serological household surveys. Lancet Glob Health. 8(11):e1390-e13e8

8. Dong Y, Mo X, Hu Y, Qi X, Jiang F, Jiang Z, et al. (2020) Epidemiology of COVID-19 among children in China. Pediatrics 145(6).

9. Minotti C, Tirelli F, Barbieri E, Giaquinto C, Donà D (2020) How is immunosuppressive status affecting children and adults in SARSCoV-2 infection? A systematic review. J Infect. 81(1):e61-ee6

10. Jamilloux Y, Henry T, Belot A, Viel S, Fauter M, El Jammal T et al (2020) Should we stimulate or suppress immune responses in COVID-19? Cytokine and anti-cytokine interventions. Autoimmun Rev. 19(7):102567

11. Prata-Barbosa A, Lima-Setta F, Santos GRD, Lanziotti VS, de Castro REV, de Souza DC, et al. Pediatric patients with COVID19 admitted to intensive care units in Brazil: a prospective multicenter study. J Pediatr (Rio J). 2020 Aug 4:S0021-7557(20)301923. https://doi.org/10.1016/j.jped.2020.07.002. Epub ahead of print.

12. Lisboa Bastos M, Tavaziva G, Abidi SK, Campbell JR, Haraoui LP, Johnston JC et al (2020) Diagnostic accuracy of serological tests for covid-19: systematic review and meta-analysis. Bmj. 370:m2516

Publisher's note Springer Nature remains neutral with regard to jurisdictional claims in published maps and institutional affiliations.

\section{Affiliations}

\section{Thaís Lira Cleto-Yamane ${ }^{1,2}$ (D) - Gustavo Rodrigues-Santos ${ }^{3} \cdot$ Maria Clara de Magalhães-Barbosa $^{3}$. Patrícia Gomes Moura ${ }^{4}$. Rafael Dias Vasconcelos ${ }^{5}$ • Jaqueline Leal Santos Gouveia ${ }^{1,6}$. Anne Louise de Oliveira ${ }^{1,6}$. Fernanda Couto Ferreira ${ }^{5}$ - Ana Letícia Shalders ${ }^{4}$ - Mariana Barros Genuíno de Oliveira ${ }^{3} \cdot$ Fernanda Lima-Setta $^{3}$. Antonio José Ledo Alves da Cunha ${ }^{3,7}$. Arnaldo Prata-Barbosa ${ }^{3,7}$}

1 Department of Kidney Transplant, Hospital Estadual da Criança, Rua Luiz Beltrão, 147, Vila Valqueire, Rio de Janeiro, RJ 21330320, Brazil

2 Department of Nephrology, Hospital Universitario Pedro Ernesto, Universidade do Estado do Rio de Janeiro, Rio de Janeiro, RJ, Brazil

3 Department of Pediatrics, Instituto D'Or de Pesquisa e Ensino (IDOR), Rio de Janeiro, RJ, Brazil
4 Department of Oncology, Hospital Estadual da Criança, Rio de Janeiro, RJ, Brazil

5 Department of Liver Transplant, Hospital Estadual da Criança, Rio de Janeiro, RJ, Brazil

6 Department of Pediatric Nephrology, Hospital Federal de Bonsucesso, Rio de Janeiro, RJ, Brazil

7 Universidade Federal do Rio de Janeiro, Rio de Janeiro, RJ, Brazil 